Research Article

\title{
Topological Design of a Mortar Base Plate under Impact Loads
}

\author{
Fengfeng Wang $(\mathbb{D})$ and Guolai Yang $\mathbb{D}$ \\ School of Mechanical Engineering, Nanjing University of Science \&Technology, Nanjing 210094, China \\ Correspondence should be addressed to Guolai Yang; yangglnjust@gmail.com
}

Received 2 September 2020; Revised 22 January 2021; Accepted 4 February 2021; Published 15 February 2021

Academic Editor: Zhixiong Li

Copyright ( $\odot 2021$ Fengfeng Wang and Guolai Yang. This is an open access article distributed under the Creative Commons Attribution License, which permits unrestricted use, distribution, and reproduction in any medium, provided the original work is properly cited.

\begin{abstract}
In this work, the topology optimization of a mortar base plate is analysed under impact loading conditions. Usually, in this case, only ordinary topology optimization under volume constraints is considered. However, to reduce the quality of the mortar base plate and facilitate engineering applications, the topological optimization problem of the continuum of the base plate under the engineering and quality constraint is considered. A finite element model has been established and verified by testing for the mortar base plate. The variable density method was used to obtain the topological optimization results of the base plate based on the force transmission path, and then the structure was reconstructed. The mass of the optimization model of the base plate is $12.78 \%$ lower than that of the original model. In comparison with the original base plate before optimization, the results show that the maximum deformation and stress of the base plate decreased by $16.85 \%$ and $35.52 \%$, respectively. Also, the firing stability of the mortar meets requirements, which not only meet the design requirement but also provide a reference for the performance improvement and structural optimization design of the base plate.
\end{abstract}

\section{Introduction}

The mortar is a curved-fire gun with the base plate bearing the recoil force, which is an important composition of the ground suppression firepower due to its features of ballistic bending, simple structure, light weight, user-friendly operation, and rapid firing response. A base plate, as an important composition of the mortar, is equivalent to a special counter-recoil device with soil as its working medium [1]. The launch load of a mortar is a strong shock load, Figure 1 shows the pressure curve at the bottom of the barrel bore by the base plate, and the action time is $6.3 \mathrm{~ms}$ with the maximum load being $112 \mathrm{MPa}$. Therefore, the mass of the base plate is heavy for guaranteeing the firing stability. What is worse, the base plate could be damaged easily after several times of firing. Achieving the lightweight design of the base plate and improving its strength and firing stability are problems to be solved urgently.

Structural topology optimization mainly includes analytical and numerical methods. Shao [2] studied the application of structural optimization technology in the light weight of the artillery structure through the analysis and calculation of the multiobjective optimization model of the gun rear frame. Dunning and Kim [3] deduced the sensitivity under uncertain load through the analysis of precise formulas and applied the level set topology optimization method to study the influence of variance in robust compliance optimization. Jang et al. [4] proposed a dynamic response topology optimization method using equivalent static loads, which generates the same displacement field as the dynamic load in each time step and then uses the static topology optimization results in dynamic analysis. Gomez and Spencer [5] and Lee and Park [6] studied the structural dynamic topology optimization under equivalent static loads. Zhao et al. [7] studied the structural topological optimization with dynamic fatigue constraints subject to dynamic random loads. Chun et al. [8] studied systemreliability-based design and topology optimization of structures under constraints on first-passage probability. Pydimarry et al. [9] studied the topological design of structures under dynamic periodic loads. James [10] and Luo et al. [11] studied the structural topology optimization under multiple working conditions and multiple objectives. Considering the discretized zero-one continuum topology 
optimization problem, Anitescu et al. [12] proposed a material interpolation model based on a certain rational function and found the optimal distribution of two linearly elastic materials such that compliance is minimized.

As a crucial optimization method in structural design, topology design has been extensively used in a variety of domains. Noh and Yoon [13] studied the topology optimization of piezoelectric energy harvesting devices considering static and harmonic dynamic loads. Yan et al. [14] studied the topology optimization of damping layers in shell structures subject to impact loads for minimum residual vibration. Dong Shin et al. [15] used the topology optimization in the lightweight design of vehicle mounted voltage converter. Amory Martin and Gregory [16] studied the structural topology optimization of tall buildings for dynamic seismic excitation using modal decomposition. Lakshmi Srinivas and Arshad [17] studied the topology optimization of industrial manipulatorlink considering dynamic loading. Ghasemi et al. [18] present a design methodology based on a combination of isogeometric analysis (IGA), level set, and point-wise density mapping techniques for topology optimization of piezoelectric/flexoelectric materials.

However, there are still few related studies. Ma [19] and Wang [20] studied the finite element analysis method and the structural optimization design method by placing the mortar base plate on different ground surfaces with the application of the nonlinear finite element theory and the structural optimization design theory. Zhang et al. [21] completed the improved design of the topology optimization of the mortar base plate through establishing a finite element model based on the OptiStruct software platform in line with the stress condition of the mortar base plate. In the design of the base plate structure, Sayir et al. [22] used multiple outwardly extending metal ribs to uniformly transfer the cyclic impact load from the anchor to the entire base plate structure.

Moreover, there are still some shortcomings in these studies. First, they did not conduct a confirmatory test on the established model. Second, in the optimization model used in topology optimization, most of the constraints considered are volume constraints. For the lightweight design of the base plate, quality constraints should be considered; as an engineering application component, the base plate must also be combined with engineering constraints; therefore, considering the quality constraints, engineering constraints, and topological issues of continuum optimization at the same time is the key to solving the optimization problem of base plates. Finally, for the performance analysis of the optimized rear base panel, there are very few studies that comprehensively consider the quality, stiffness, strength, and shooting stability. Therefore, this manuscript will combine relevant researches to focus on the above deficiencies and carry out the topology optimization research of the mortar base plate.

\section{Topological Design of a Mortar Base Plate}

The topological design process of the mortar base plate is shown in Figure 2.
2.1. Modeling. Focusing on a trapezoidal pyramid base plate at the caliber of $120 \mathrm{~mm}$, the main plate is comprised of three trapezoidal thin plates distributing symmetrically at $120^{\circ}$ via welding. There is a cone-shaped basin in the middle of the main plate, while there is strengthened flanging around the main plate. A stationary joint is welded at the center of the cone-shaped basin; and there are three sets of large and small studs inside the base plate. The external part of the stud connected with the wrapped rib; and the spade is linked at the bottom of the wrapped rib and the stationary joint. The whole base plate is made of TC4 titanium alloy with a mass of $66.5 \mathrm{Kg}$. The structure of the reinforcing plate of the base plate is presented in Figure 3.

Finite element models of the original structure of the base plate and the exposure to soil have been established with the use of hypermesh. Partial views apart from the main base plate and the cone-shaped basin and the internal rib plate are presented in Figure 4. According to the structural characteristics of the base plate, a hybrid grid element based on quadrilateral grid elements is used, and the parts are connected by common nodes. When the flow field trend is basically correct, the grid is gradually refined, and the results of multiple calculations are compared; and a grid model with good convergence, fast calculation speed, and relatively accurate grid is obtained, as shown in Table 1. In the established model, the total number of elements in the finite element model of the base plate is 23650, and the total number of nodes is 22367. The material parameters of the base plate and soil are shown in Tables 2 and 3, respectively.

The interaction relationship between the tail ball and the mortar is simulated through surface-to-surface contact, and the elevation angle changes of the mortar are simulated by adjusting the angle of the tail ball. The impact load is applied to the breech end face, as shown in Figure 5.

The contact surface between the base plate and the soil is set as a fixed constraint upon importing the established finite element model into calculation. Then, the stress condition of the base plate is presented in Figure 6 with the addition of the launching load in the stationary joint center. The stress of the base plate is mainly distributed around the stationary joint and at the intersection of the main plate and the coneshaped basin. They are, respectively, at A and B in Figure 6. What is more, the base plate is easily deformed or damaged in these two areas due to the significant stress concentration in these areas, thus affecting the strength and stability of the whole structure. Therefore, it is of great necessity to improve the stress distribution and the stress concentration of the base plate through optimizing the design method.

2.2. Model Verification and Analysis. $n$ impact mechanical performance test system for the mortar base plate is developed to verify the correctness of the model, which simulates the launching of load with the electromagnet to control the drop-hammer impact. The system consists of a test bench, medium-hard soil samples, a base plate, test sensor, and data acquisition system. Test schematic diagram and the test are shown in Figures 7 and 8. Two points with the maximum stress of the base plate or point $\mathrm{A}$ around the 


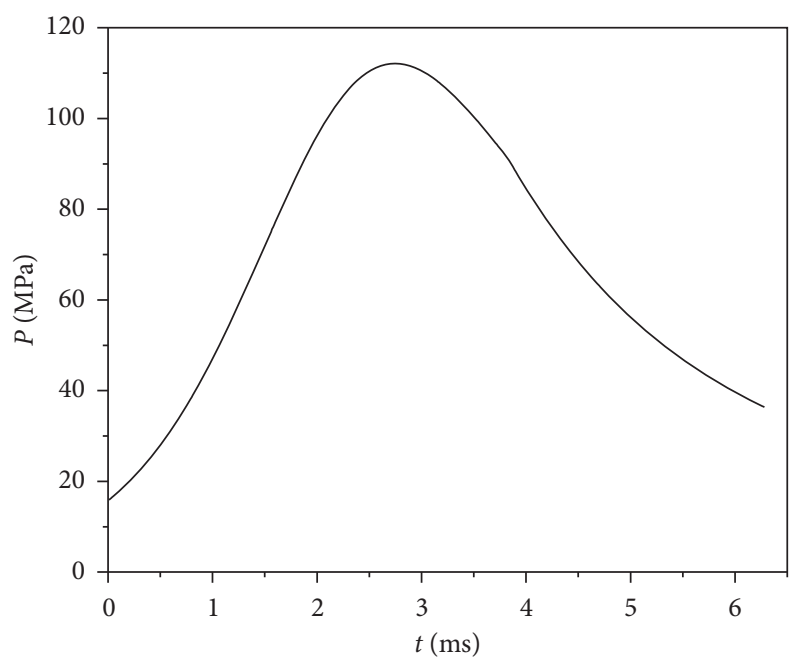

Figure 1: Bore bottom pressure curve.

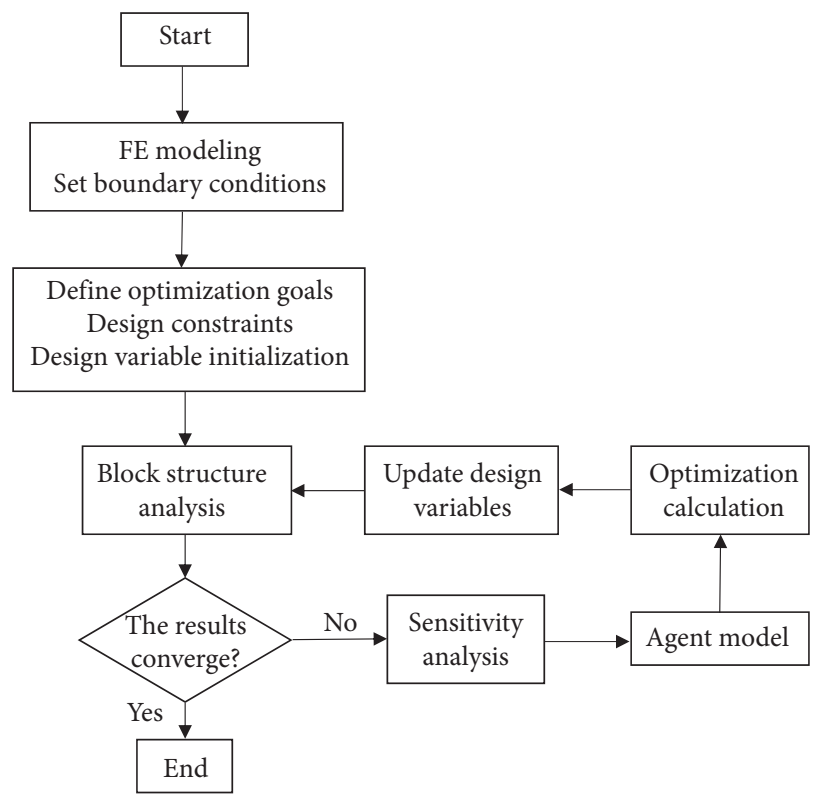

Figure 2: Topology design process of a mortar base plate.

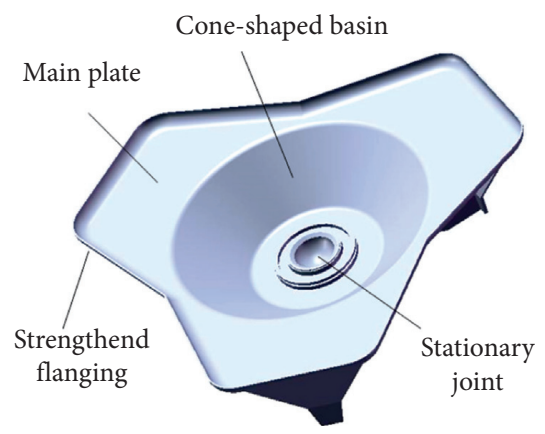

(a)

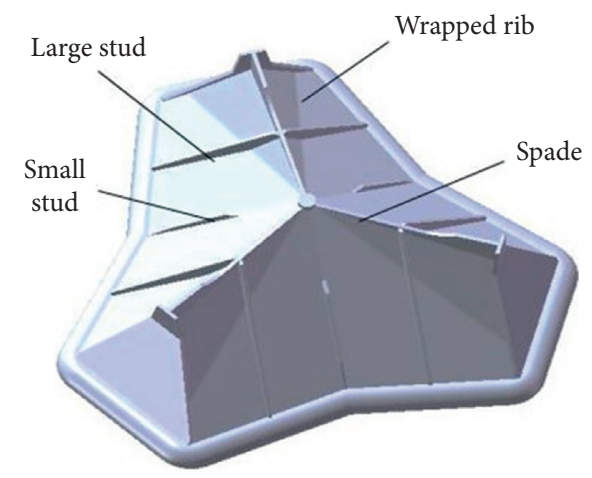

(b)

FigURE 3: Structure of base plate. (a) Front side. (b) Back side. 


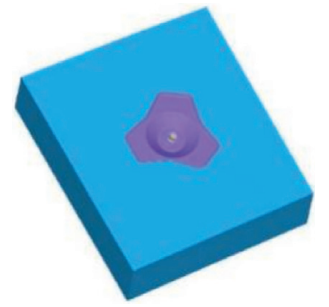

(a)

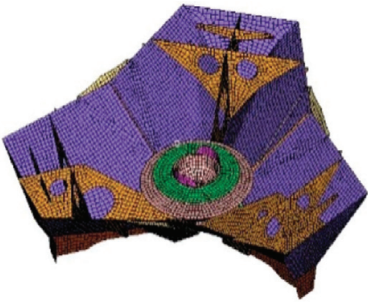

(b)

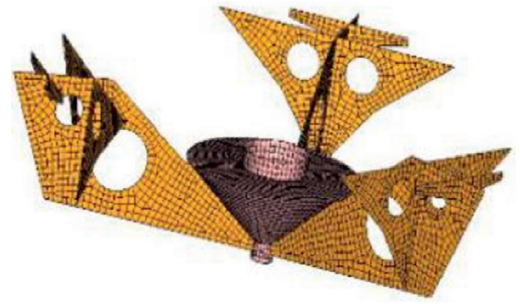

(c)

Figure 4: Original structure of base plate. (a) Finite element model of plate and soil contact. (b) Partial view. (c) Internal ribs.

TABLE 1: Comparison of calculation results of sparse and dense models.

\begin{tabular}{lcccc}
\hline Model & Number of grids & Maximum stress $(\mathrm{MPa})$ & Maximum displacement $(\mathrm{mm})$ & Relative time $(\mathrm{s})$ \\
\hline 1 & 9983 & 239.8 & 3.23 & 2060 \\
2 & 18551 & 286.9 & 3.98 & 2345 \\
3 & 23650 & 333.3 & 4.303 & 2576 \\
4 & 34280 & 339.5 & 4.301 & 3960 \\
\hline
\end{tabular}

TABLE 2: Material parameters of titanium alloy.

\begin{tabular}{lccc}
\hline Grade & Density $\left(\mathrm{t} \cdot \mathrm{mm}^{-3}\right)$ & Elastic modulus (MPa) & Poisson's ratio \\
\hline TC4 & $4.51 \times 10^{-9}$ & $1.0 \times 10^{5}$ & 0.34 \\
\hline
\end{tabular}

TABLE 3: Material parameters of soil.

\begin{tabular}{lccccc}
\hline Elastic modulus E/MPa & Poisson's ratio $\mu$ & Density $\rho /(\mathrm{t} \cdot \mathrm{mm}-3)$ & Friction angle $\beta /^{\circ}$ & Yield stress ratio K & Expansion angle $\psi /^{\circ}$ \\
\hline 100 & 0.29 & $2 \times 10^{-9}$ & 28 & 0.911 & 10 \\
\hline
\end{tabular}

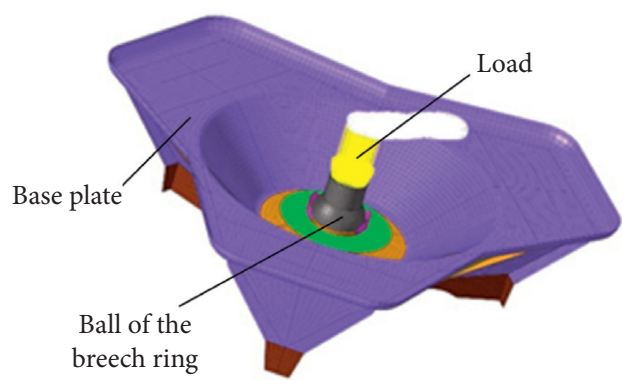

FIgURE 5: Applying load.

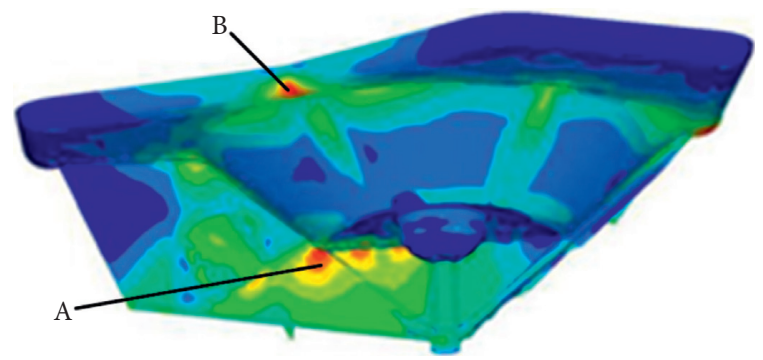

FIGURE 6: Stress situation of base plate.

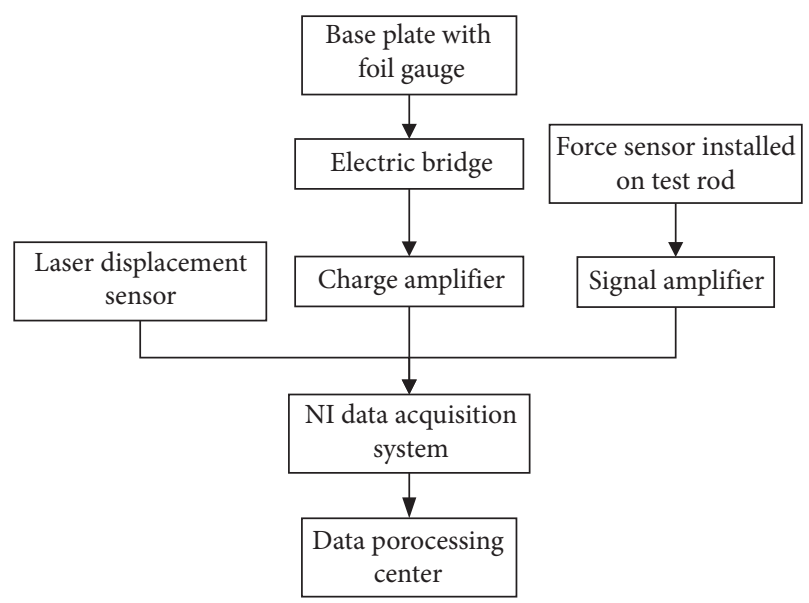

FIgURE 7: Test schematic diagram.

stationary joint and point $B$ at the intersection of the main plate and the cone-shaped basin in Figure 3 are selected as measuring points.

Within consideration of some of the influencing factors during the drop weight experiment, the attenuation coefficient $\mathrm{K} 1$ is introduced into the impact load calculation formula (1) to obtain 


$$
\mathbf{F t}=\mathrm{mvK}_{1},
$$

where $F$ is the average force; $t$ is the impact time; $m$ is the falling weight; $v$ is the impact velocity of the falling weight; $\mathrm{K} 1$ is the attenuation coefficient, which is 0.7 in this test.

It is known that the falling weight is $300 \mathrm{~kg}$. In the test, the falling weight is raised to the specified height. According to the law of conservation of energy, formula (2) can be obtained:

$$
\mathrm{mgh}=\frac{1}{2} \mathrm{mv}_{1}^{2},
$$

where $g$ is $9.8 \mathrm{~m} / \mathrm{s} 2$. The impact velocity $\mathrm{v} 1$ of the falling weight is calculated by formula (2).

Within consideration of the air resistance during the fall, the calculation formula of the attenuation coefficient $\mathrm{K} 2$, which is 0.9 in this test, is given as follows:

$$
v=K_{2} v_{1} \text {. }
$$

According to the test design, the impact test of the mortar base plate was completed. The impact was applied at an interval of $3 \mathrm{~cm}$ from the distance between the electromagnet and the loading platform. The maximum load force measured by the force sensor was $50300 \mathrm{~N}$, and the duration of action was $50 \mathrm{~ms}$. After conversion, the impact load is 100.12 $\mathrm{MPa}$, which is close to the theoretical value.

By comparing test results with the simulation results under the same working condition, the strain comparison of the two measuring points is shown in Figure 9.

As can be seen from Figure 9, the position and dimension of the maximum simulation value and experimental value curve are basically consistent with similar change trends. Meanwhile, the correctness of the established simulation model can be verified initially, providing a simulation approach for structural design and optimization of the base plate.

2.3. Optimization Process and Results. In this research, the dynamic topology optimization problem of continuum structure of mortar plate under engineering constraints is studied. The topology optimization of the continuum structure adopts the finite element method to discretize the optimized design area structure to form a limited number of units, and each unit corresponds to a design variable. The design variables and stiffness of the finite element are processed and calculated based on the finite element method for the original structure [23].

The commonly used material interpolation models in topology optimization include SIMP (solid isotropic material with penalization) material interpolation model and RAMP (Rational Approximation of Material Properties) material interpolation model [24]. The introduction of continuous variables through the SIMP material interpolation method can solve the discontinuous problem of discrete variables in topology optimization. The SIMP threedimensional material interpolation formula used in this research is

$$
E_{e}\left(x_{e}\right)=E_{\min }+x_{e}^{p}\left(E_{o}-E_{\min }\right), \quad x_{e} \in[0,1],
$$

where $x_{e}$ represents unit design variables (i.e., relative density); $p$ represents penalty index; $E_{e}$ represents the element elastic modulus corresponding to the element design variable $x_{e}$ (the element elastic modulus after interpolation and update); $E_{o}$ represents the modulus of elasticity of the original material; $E_{\min }$ represents hollow material elastic modulus, generally 0.001 times of $E_{o}$.

The optimized mathematical model designed in this paper can be described as

$$
\begin{aligned}
& \text { Find } x=\left(x_{1}, x_{2}, \ldots, x_{n}\right)^{T}, \\
& \operatorname{Min} C(x)=F^{T} U, \\
& \text { S.t. }\left\{\begin{array}{l}
M_{2} \leq M_{1}, \\
S_{2} \leq S_{1}, \\
F=\mathrm{KU}, \\
0<x_{\min }<x_{i}<1, \quad(i=1,2, \ldots, n),
\end{array}\right.
\end{aligned}
$$

where $x_{i}$ is a design variable with the continuous value taken from $\left[x_{\min }, 1\right]$ (0 represents the deletion of the unit, and 1 represents the retention of the unit); $n$ is the number of optimized design variables. The other structure of the base plate except the spade, large stud, and small stud is used as a design space in this research, and the element density in the design space is used as the design variable; $K$ is the total stiffness matrix of the structure; $U$ is the displacement vector of the structure; $F$ is the structural force vector; $M_{1}$ is the initial mass before structural optimization; and $M_{2}$ is the mass after structural optimization; $S_{1}$ is the maximum stress before topology optimization; and $S_{2}$ is the maximum stress after topology optimization.

The topological optimization of the finite element model of the base plate is solved. The optimized result of the base plate can be obtained, and it converged after 43 iterations, as shown in Figure 10. It can be seen from the figure that the stationary joint receiving the force transmitted directly from the gun breech's ball is the first load-bearing area; and the spherical surface of the stationary joint transferring the force to the whole base plate with the force transmitted to the cone-shaped basin being transmitted to the main plate along with the path of three studs intersecting with the coneshaped basin is the second load-bearing area. The area transmitting the force to the large stud and transmitting externally to the small perpendicularly intersected stud via the wrapped rib through the large stud is the third loadbearing area. The area transmitting the force to the spade at the bottom and then to the soil is the fourth load-bearing area; and the remaining transparent areas are out of main load-bearing areas.

2.4. Optimal Design of the Plate Structure. Materials on the force transmission path are guarantees of the bearing capacity of the base plate, all of which must be reserved in the optimized design. Materials of other parts with small stress can be appropriately removed. The CAE model upon 
topology optimization is imported into the CAD software for redesigning the structure of the base plate based on the CAD/CAE interface technology. The geometric model of the redesigned base plate is presented in Figure 11.

Gridding is conducted on the established geometric model of the base plate by setting the material property as titanium alloy to complete the finite element modeling of the base plate. The finite element model of the rear sill is optimized. Partial views apart from the main base plate and the cone-shaped basin of the finite element model of the optimized base plate and the internal rib plate are presented in Figure 12. Ribs are continuously contacted to ensure a complete force transmission path. The mass of the base plate model upon topology optimization is changed from $66.5 \mathrm{~kg}$ to $58 \mathrm{~kg}$, by comparison with the original model. The mass of the optimization model of the base plate is $12.78 \%$ lower than that of the original model.

\section{Results and Discussion}

3.1. Modal Comparison of the Base Plate. Modal analysis is performed on the original model and the optimization model. As the free modal calculation is set, the first-order modal result is shown in Figure 13. The first-order modal frequency of the original model is $369.83 \mathrm{~Hz}$ with the modal deformation of $1.217 \mathrm{~mm}$. The first-order modal frequency of the optimization model of the base plate is $379.90 \mathrm{~Hz}$ with the modal deformation of $1.222 \mathrm{~mm}$. The first-order modal frequency of the optimization model of the base plate is merely increased by $2.72 \%$ in comparison with the original one. Because the frequencies useful for engineering design are mainly concentrated in the low-order parts, the first 5 order frequency comparisons are added. The comparison values are shown in Table 4 . No significant change can be witnessed from the vibration characteristics upon optimization.

3.2. Strength Comparison of the Base Plate. The firing working condition is at the direction angle of $0^{\circ}$ and at the elevation angle of $75^{\circ}$. The original model and the optimization model of the base plate are simulated separately for calculation in the firing simulation.

The stress cloud diagram and the displacement cloud diagram of the original model of the front and back sides of the original model and the optimization model of the base plate are presented in Figures 14 and 15, respectively. As can be seen from the figures, the maximum stresses of the optimization model and the original model are $214.9 \mathrm{MPa}$ and $333.3 \mathrm{MPa}$, respectively. The maximum stress of the optimization model is decreased by $35.52 \%$ compared with the original model. Meanwhile, no significant stress concentration can be observed on the surface of the optimization model.

The maximum deformations of the optimization model and the original model are $3.578 \mathrm{~mm}$ and $4.303 \mathrm{~mm}$, respectively. The maximum displacement of the optimization model is decreased by $16.85 \%$ compared with the original model, indicating that the deformation of the optimization model is smaller than that of the original model with strength higher than that of the original model. Therefore, the structural performance of the original model is enhanced. The large deformation of the optimization model is distributed merely on one side near the stationary joint, which is related to the set working conditions upon analysis. As the deformations on both sides have the same order of magnitude with a small difference, they are within the design tolerance range.

In order to comprehensively measure the strength of the base plate before and after optimization, this paper also compares the three working conditions of $0^{\circ} / 85^{\circ}, 60^{\circ} / 75^{\circ}$, and $60^{\circ} / 85^{\circ}$ and synthesizes the finite element values of the four working conditions. The results are compared as shown in Table 5.

In the four working conditions, the change rate of the maximum stress after optimization is about $35 \%$, and the change rate of maximum displacement is about $12 \%-20 \%$. The maximum stress after optimization is significantly reduced, the maximum displacement is also greatly reduced, and the optimization effect is obvious. Regarding the phenomenon that the stress of the optimized back base plate is concentrated on the side of the socket, it is because the design area of the optimized structure is transmitted according to the force transmission path, so the maximum stress received is greatly improved. However, under a huge impact load, the force on the base plate cannot be released at the socket, and the place where the maximum stress occurs is the area where the contact surface of the breech ball is larger, and it is related to the elevation angle. It is reasonable that the optimized base plate has stress concentration on the side of the socket.

3.3. Firing Stability of the Mortar. The movement of the mortar during firing is roughly composed of accelerated recoil, decelerated recoil, and counter-recoil. The recoil stability of the fired ball out of the muzzle refers to the limited displacement of the axial movement of the base plate. The recoil stability at the counter-recoil stage refers to the limitation of the bounce and the reliability of the base plate after the recoil is in place. A kinetic model of a light mortar containing a mortar and its support frame is shown in Figure 16.

The direction of the axis of the bore of the mortar is set as the $\mathrm{Z}$-axis direction. A local coordinate system is established with the projection surface of the muzzle as the $\mathrm{X}-\mathrm{Y}$ plane in accordance with the right-hand spiral rule. The upper and lower limits of the displacement $\delta$ of the base plate in the $\mathrm{X}$-axis direction and the $\mathrm{Z}$-axis direction are shown in Figure 17. By analysing the figure, in the $\mathrm{X}$-axis direction, the displacement amplitude of the optimization model in the recoil phase is slightly smaller than that of the original model, while the displacement amplitude of the optimization model of the counter-recoil phase is basically consistent with that of the original model. In the $\mathrm{Z}$-axis direction, the displacement amplitude of the optimization model in the recoil phase is basically consistent with that of the original model, while the displacement amplitude of the 


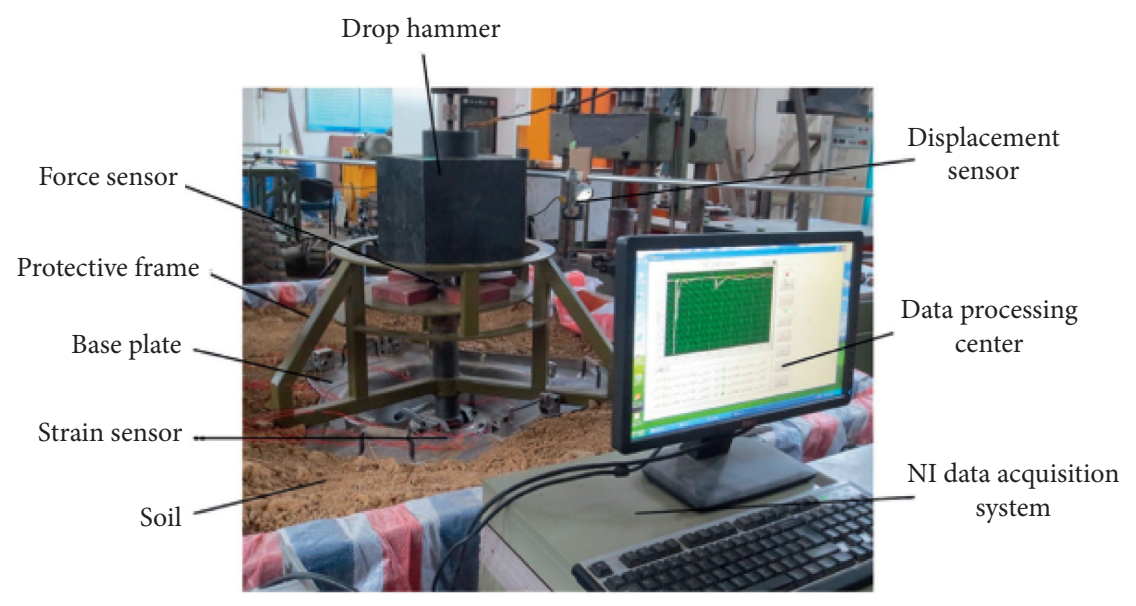

Figure 8: Test.

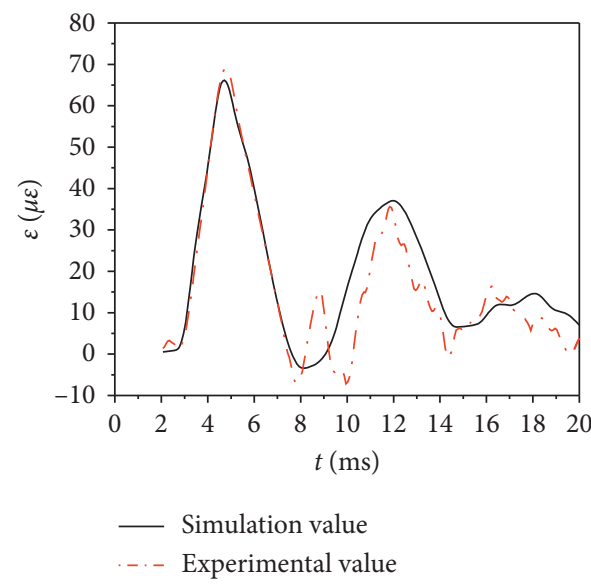

(a)

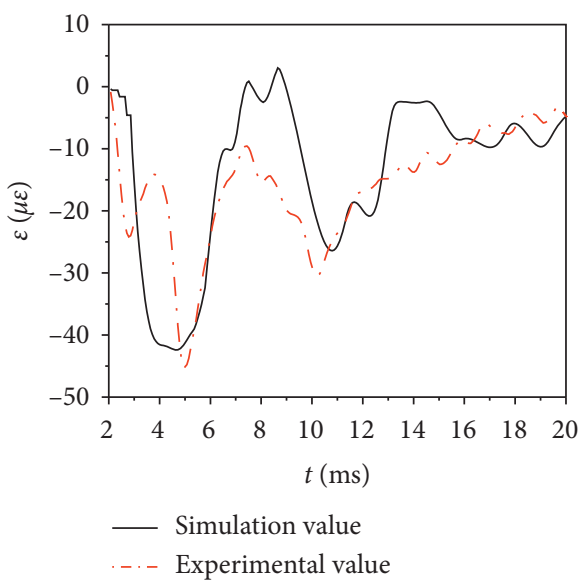

(b)

Figure 9: Comparison of test and simulation. (a) Point A. (b) Point B.

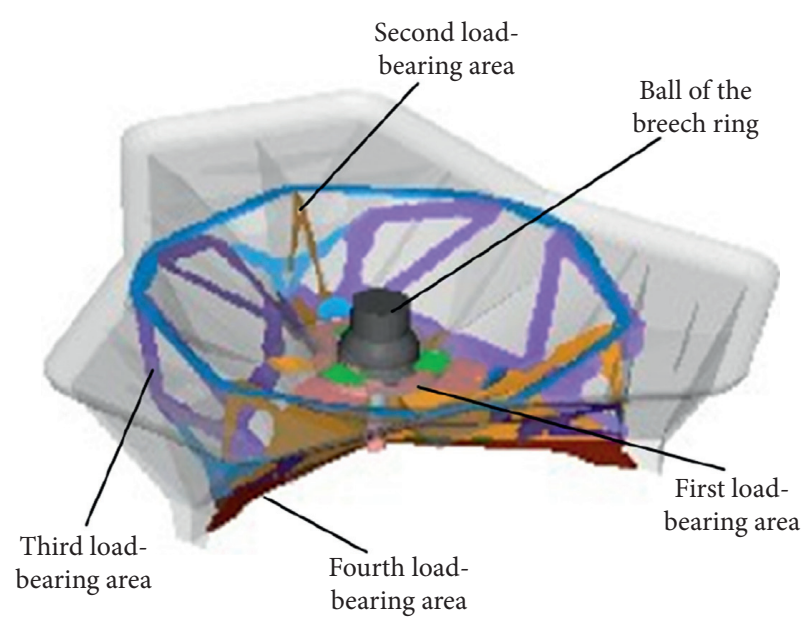

FIGURE 10: Topology optimization results of the base plate.

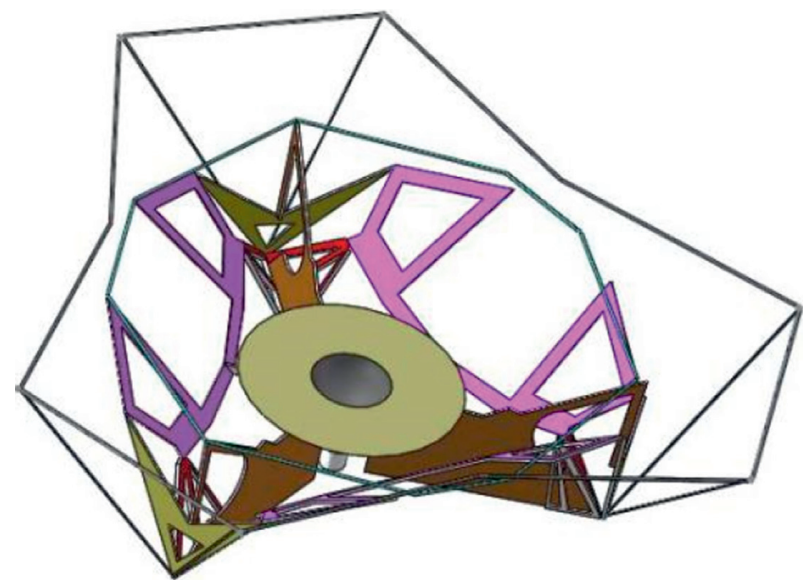

FIGURE 11: Geometric model of optimized base plate. 


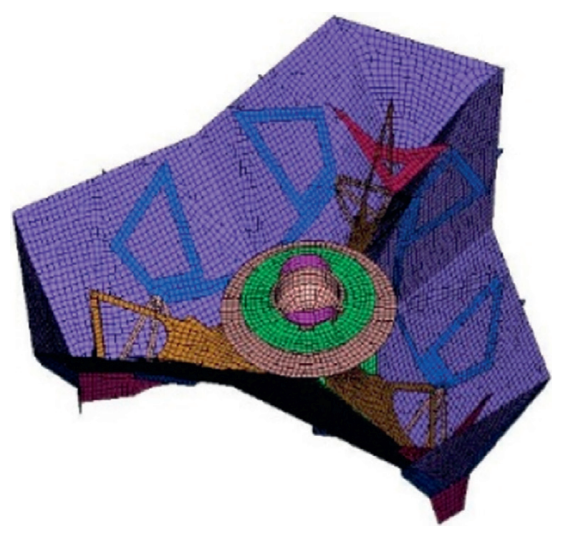

(a)

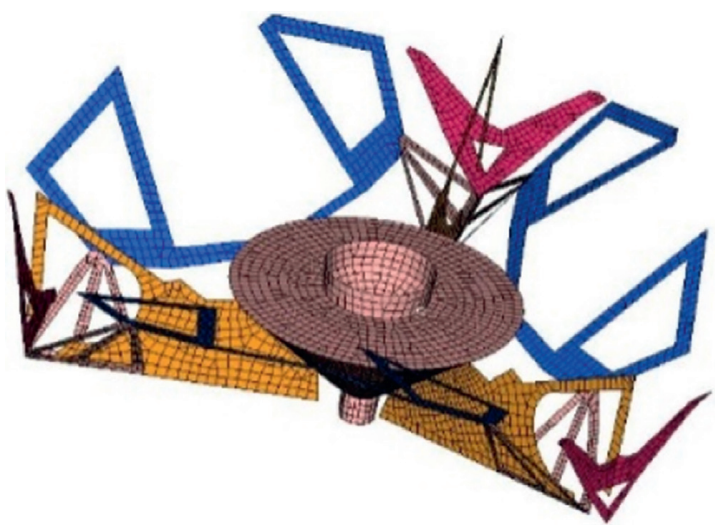

(b)

Figure 12: Finite element model of optimized base plate. (a) Partial view. (b) Internal ribs.

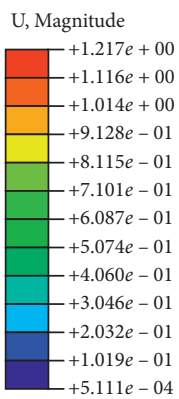

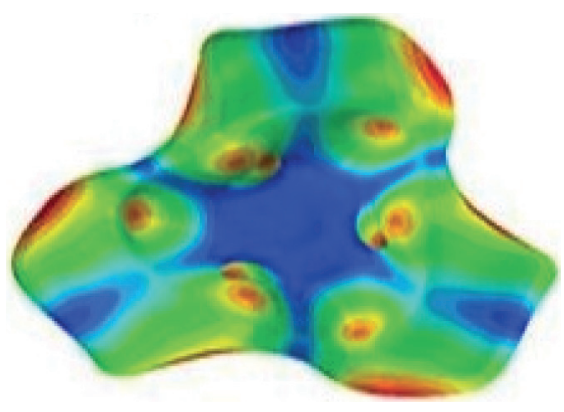

(a)

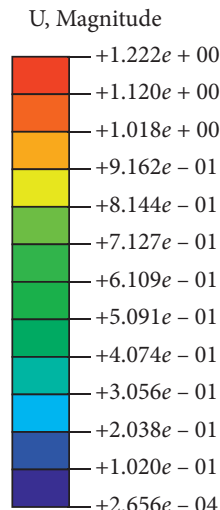

$+2.656 e-04$

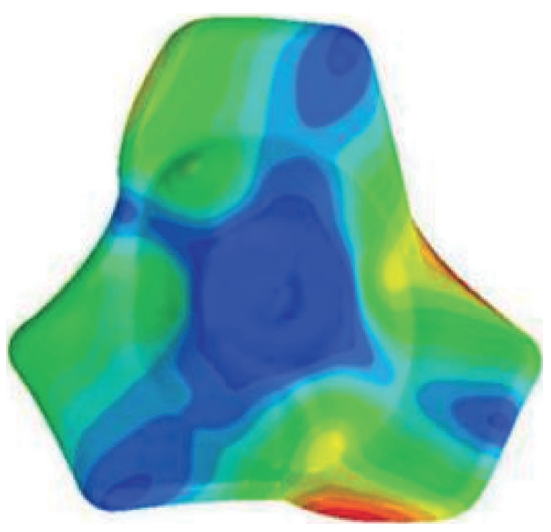

(b)

Figure 13: First-order modal comparison. (a) Original model. (b) Optimized model.

TABLE 4: Comparison of the first 5 modal values of the base plate before and after optimization.

\begin{tabular}{|c|c|c|c|c|c|}
\hline Modal order & 1st order & 2nd order & 3rd order & 4th order & 5th order \\
\hline Value of the original model $(\mathrm{Hz})$ & 369.83 & 369.90 & 477.39 & 687.78 & 689.47 \\
\hline Value of the optimized model $(\mathrm{Hz})$ & 379.90 & 379.92 & 513.41 & 714.19 & 717.25 \\
\hline Rate of change & $2.72 \%$ & $2.71 \%$ & $7.55 \%$ & $3.84 \%$ & $4.03 \%$ \\
\hline
\end{tabular}

optimization model of the counter-recoil phase is slightly smaller than that of the original model. All in all, high stability can be witnessed in the optimization model.

Moreover, the possible rotation of the gun body during mortar firing as another important factor affecting the firing stability of the mortar, in general, is measured by the displacement and the disturbance of the gun muzzle. The time for the shell acting in the bore of the mortar is $6.3 \mathrm{~ms}$ when the mortar is fired. Therefore, we mainly focus on the relevant parameter values at this moment. The displacement curve and the angular displacement curve of the gun muzzle of the original model and the optimization model of the base plate are presented in Figure 18. To be specific,
$U_{1}$ represents the vertical displacement of the muzzle, $\theta_{x}$ represents the vertical angular displacement of the muzzle, $U_{1}$ and $\theta_{x}$ present the vertical disturbance level of the muzzle, $U_{2}$ represents the horizontal displacement of the muzzle, $\theta_{y}$ represents the horizontal angular displacement of the muzzle, and $U_{2}$ and $\theta_{y}$ present the horizontal disturbance level of the muzzle.

As can be seen from Figure 18(a), the vertical displacement curve $U_{1}$ of the muzzle center of the optimization model and the original model of the base plate is increased gradually, and the vertical displacement of the optimization model is decreased by $24.65 \%$ in comparison with the original model. As can be seen from Figure 18(b), the horizontal displacement 

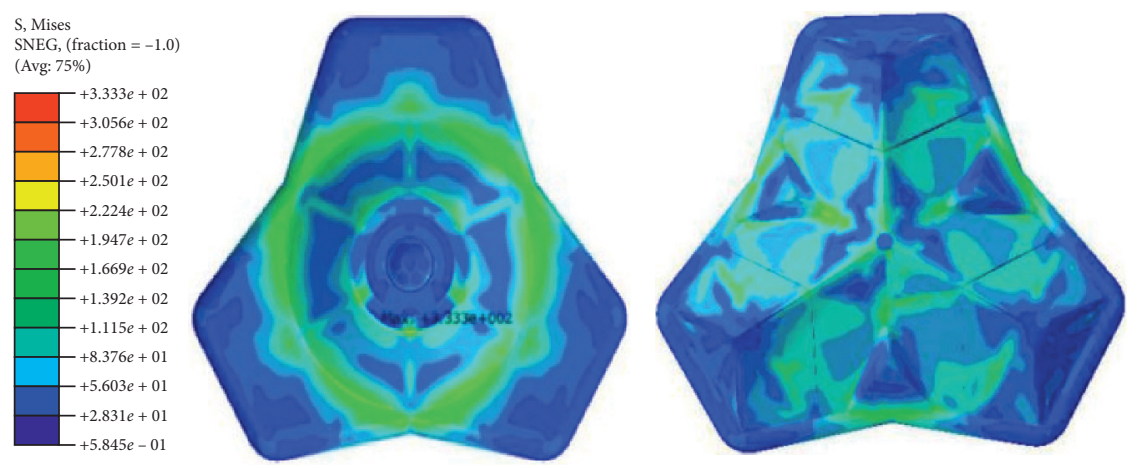

(a)
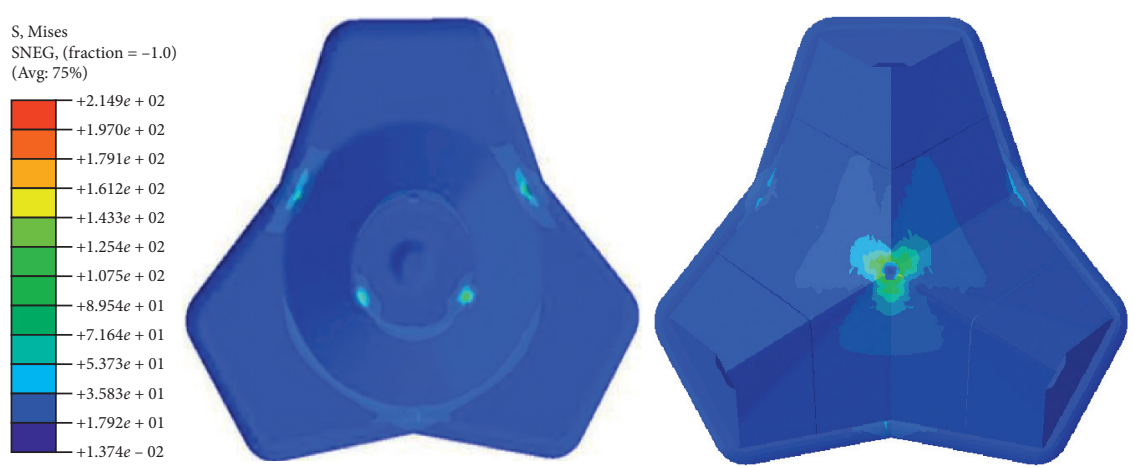

(b)

FIGURE 14: Stress nephogram of the original model and the optimized model. (a) Original model. (b) Optimized model.
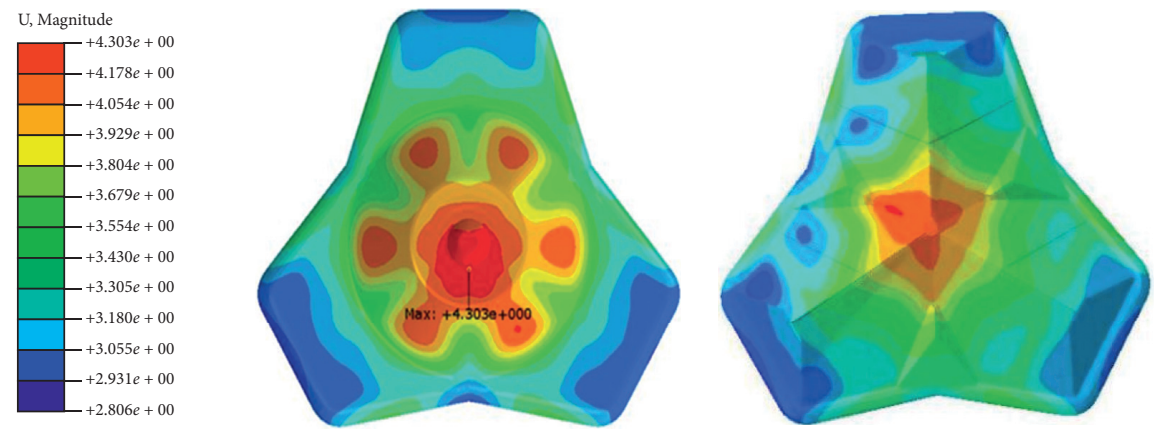

(a)
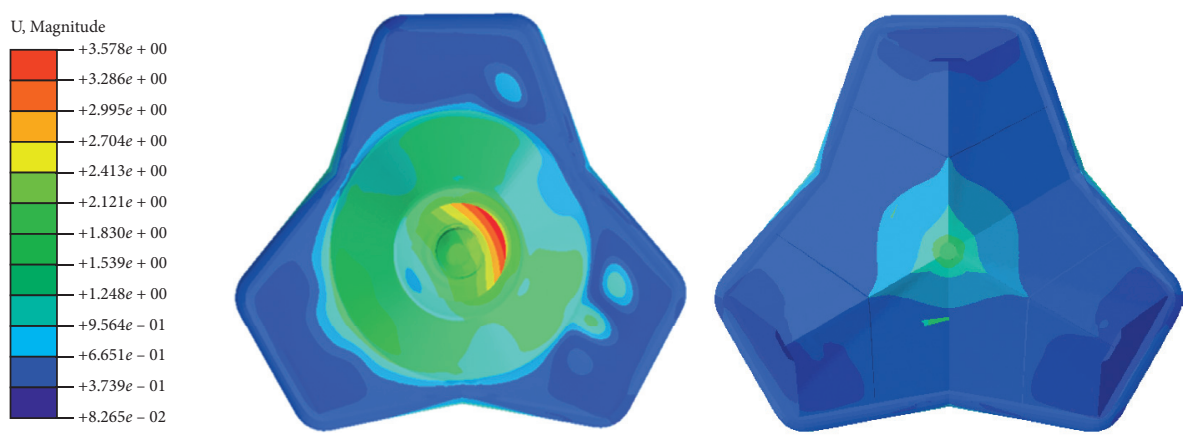

(b)

FIGURE 15: Displacement nephogram of the original model and the optimized model. (a) Original model. (b) Optimized model. 
TABle 5: Comparison of finite element numerical results of base plate before and after optimization.

\begin{tabular}{lcccccc}
\hline \multirow{2}{*}{ Working condition (direction angle/elevation angle) } & \multicolumn{3}{c}{ Maximum stress/MPa } & \multicolumn{3}{c}{ Maximum displacement/mm } \\
& Original & Optimized & Change rate & Original & Optimized & Change rate (\%) \\
\hline $0^{\circ} / 75^{\circ}$ & 333.3 & 214.9 & 35.52 & 4.303 & 3.578 & 16.85 \\
$0^{\circ} / 85^{\circ}$ & 316.3 & 203.8 & 35.57 & 4.163 & 3.555 & 14.6 \\
$60^{\circ} / 75^{\circ}$ & 325.1 & 212.7 & 34.57 & 4.30 & 3.765 & 12.44 \\
$60^{\circ} / 85^{\circ}$ & 317.8 & 206.2 & 35.12 & 4.191 & 3.378 & 19.4 \\
\hline
\end{tabular}

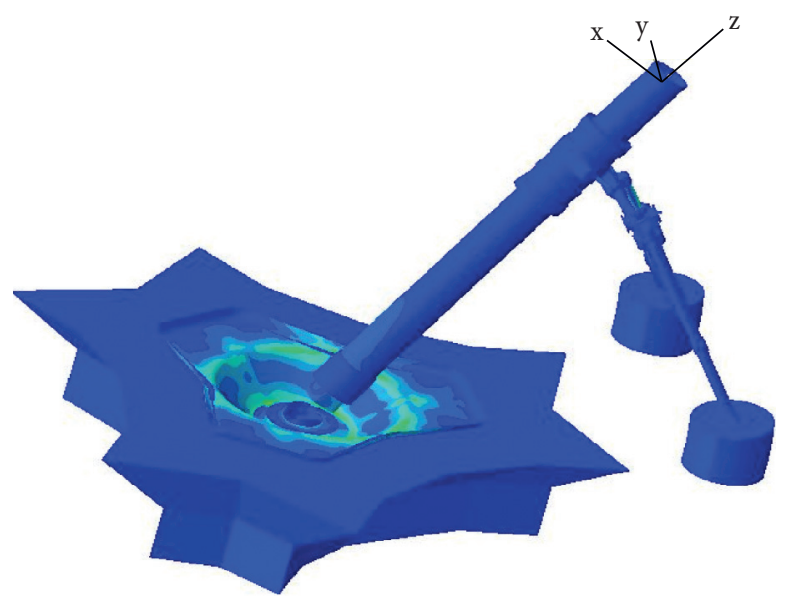

FIgURE 16: Dynamic model of mortar full gun.

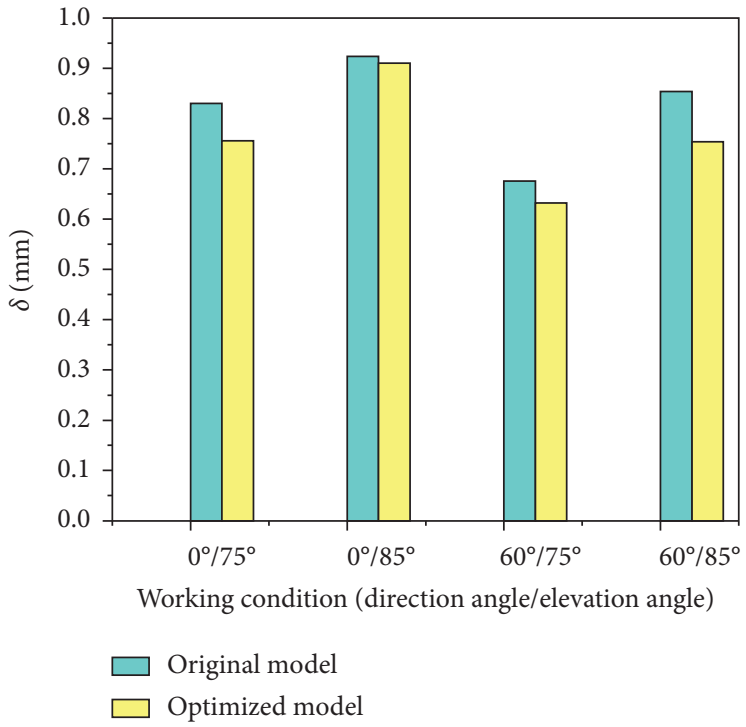

(a)

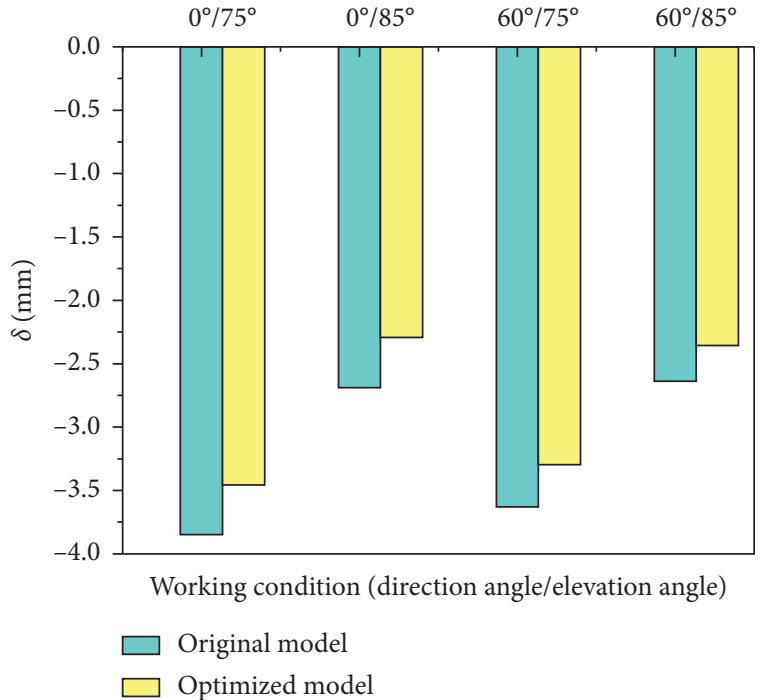

(b)

Figure 17: Continued. 


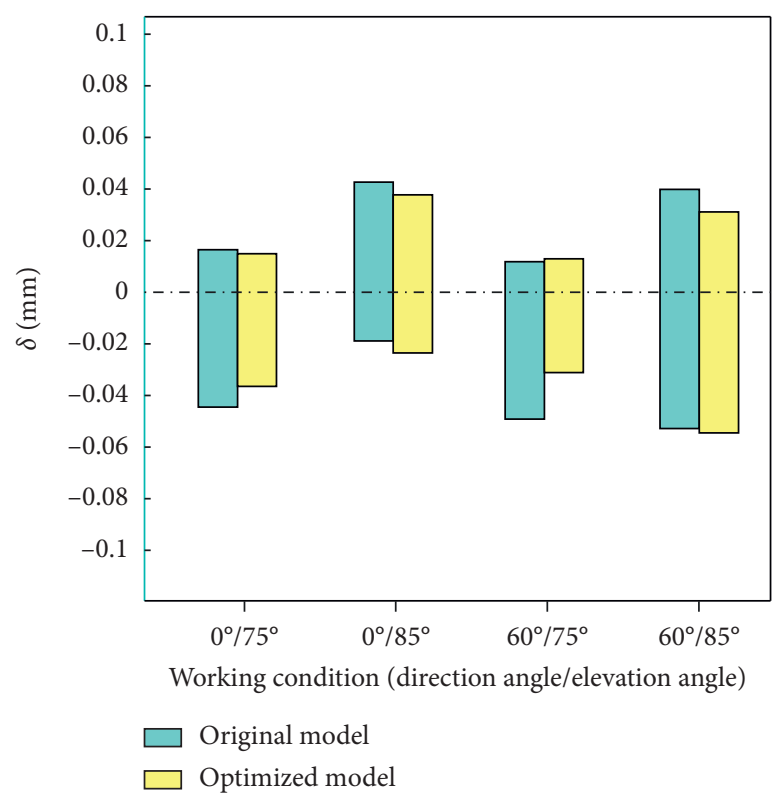

(c)

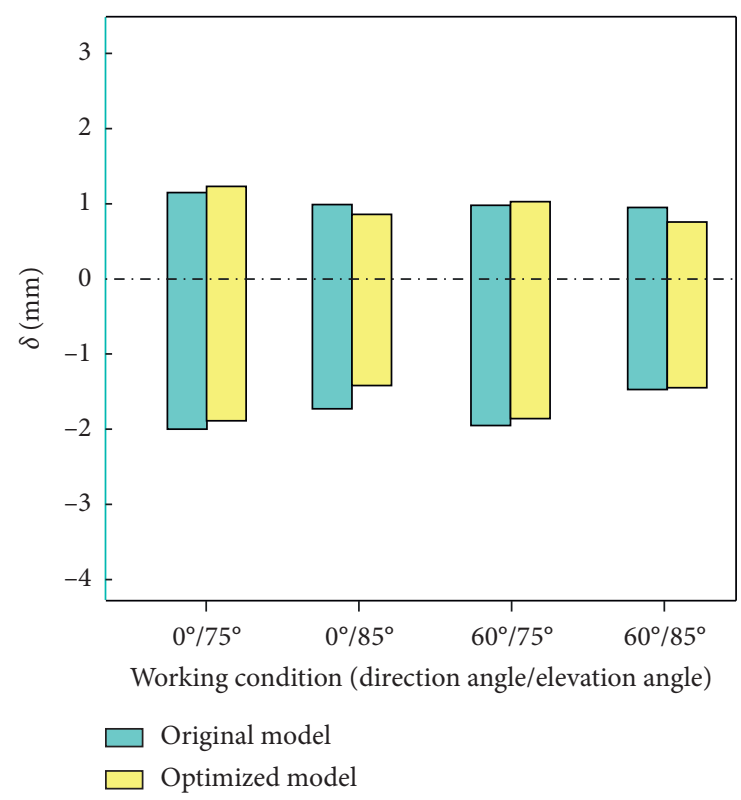

(d)

Figure 17: The comparison between the upper and lower limits of the displacement of the base plate before and after the optimization in the $\mathrm{x}$-direction and $\mathrm{z}$-direction in the recoil phase and the counter-recoil phase. (a) The upper and lower limits of the displacement in the $\mathrm{X}$-axis direction during the recoil phase. (b) The upper and lower limits of the displacement in the Z-axis direction during the recoil phase. (c) The upper and lower limits of the displacement in the X-axis direction during the counter-recoil phase. (d) The upper and lower limits of the displacement in the $\mathrm{Z}$-axis direction during the counter-recoil phase.

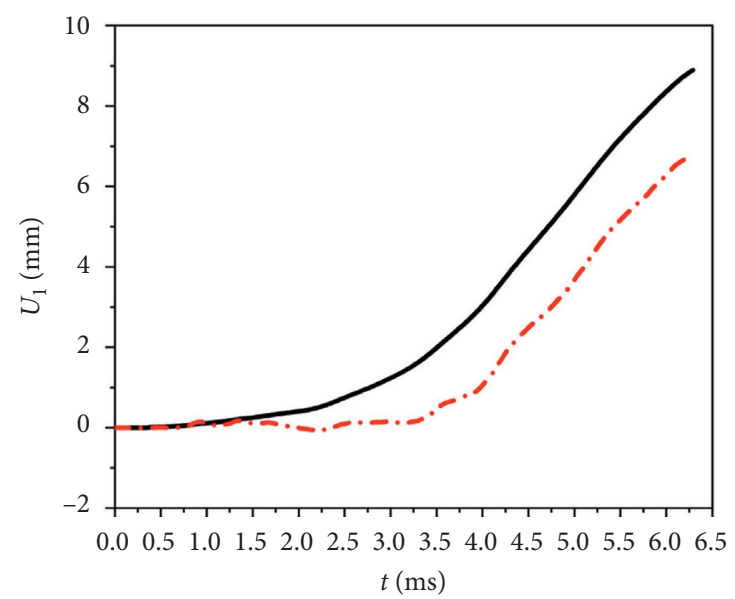

Original model

-..- Optimized model

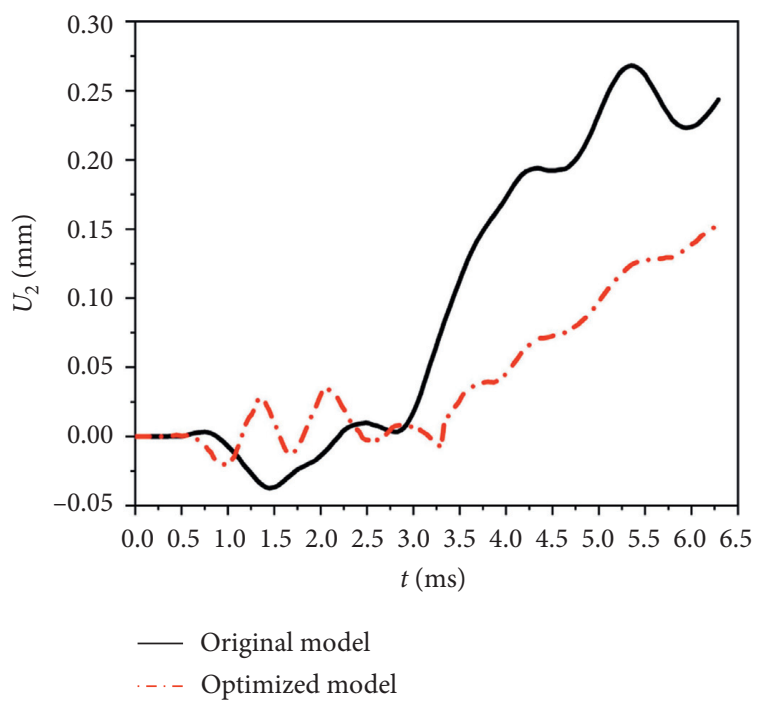

(b)

Figure 18: Continued. 


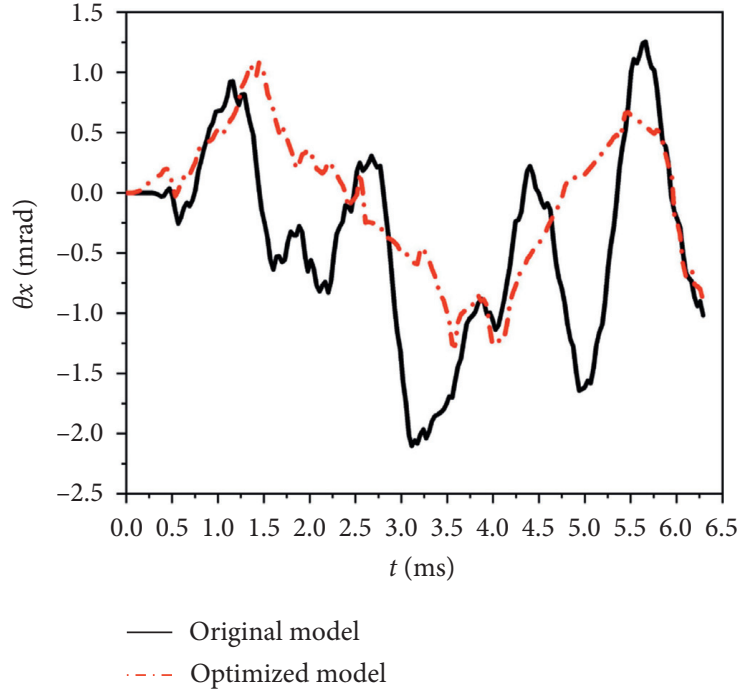

(c)

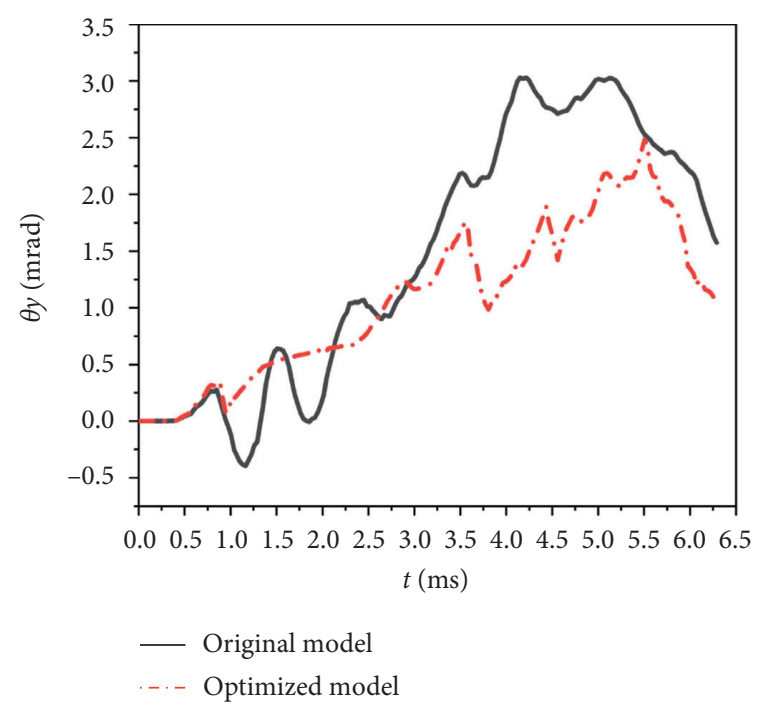

(d)

Figure 18: Muzzle winding level of the original model and the optimized model. (a) Muzzle vertical displacement curve $\left(U_{1}\right)$. (b) Muzzle horizontal displacement curve $\left(U_{2}\right)$. (c) Muzzle vertical angular displacement $\left(\theta_{x}\right)$. (d) Muzzle horizontal angular displacement $\left(\theta_{y}\right)$.

curve $U_{2}$ of the muzzle center of the optimization model and the original model of the base plate is basically consistent with a slight fluctuation at some moments, and the overall trend is increasing. More importantly, the horizontal displacement of the optimization model is decreased by $37.37 \%$ at the muzzle. As can be seen from Figure 18(c), the elevation displacement curve $\theta_{x}$ of the muzzle of the optimization model and the original model of the base plate is fluctuating up and down as a whole, with a similar change law. As can be seen from Figure 18(d), the horizontal angular displacement $\theta_{y}$ goes up first and then goes down and goes up again. The optimization model is closer to zero in comparison with the original model when the fired ball is at the muzzle whether it is vertical angular displacement $\theta_{x}$ or the horizontal angular displacement $\theta_{y}$. The disturbance embodied in the optimization model and that in the original model with a smaller disturbing quantity are similar and more approaching to 0 at the muzzle.

In order to compare the muzzle disturbance of the mortar, the numerical comparison of the horizontal and vertical displacements and angular displacements at the time when the gun ejects the muzzle under the 4 working conditions is shown in Figure 19.

As illustrated, the displacement in the recoil phase, the displacement in the counter-recoil phase, the vertical and horizontal displacement, and angular displacement of the muzzle before and after the optimization are similar overall, and the stability of the optimized base plate can be guaranteed. To sum up, the stiffness, the strength, and the stability of the base plate that is optimized in structural design based on topology optimization conform to the design requirements. Meanwhile, the structural performance of the optimized base plate has been enhanced remarkably in comparison to the original base plate, achieving the objective of design.

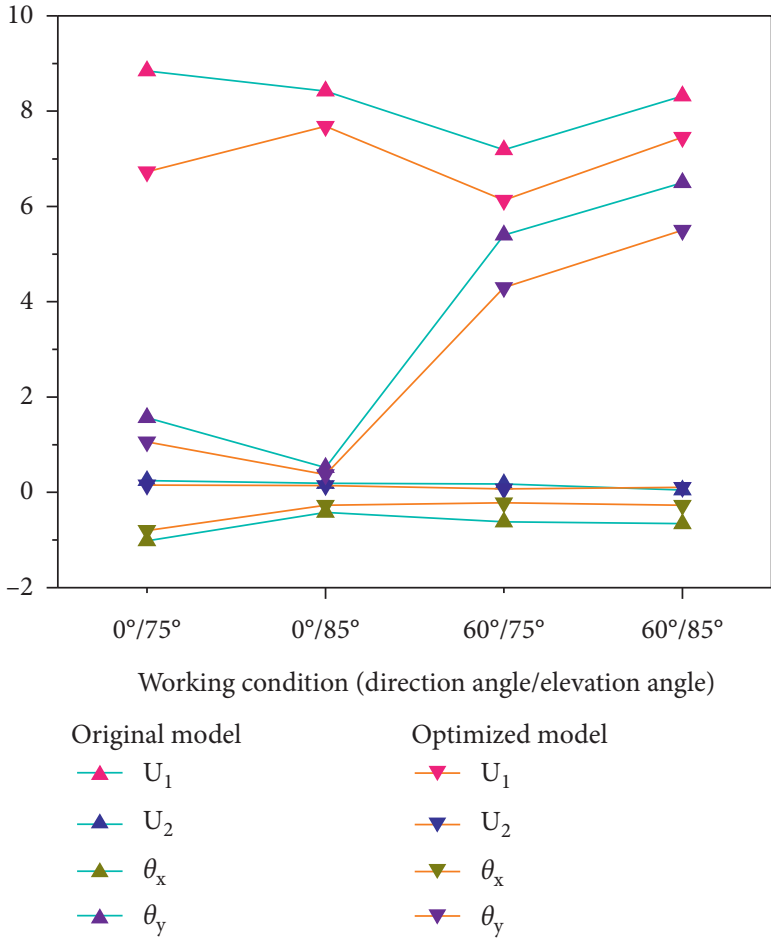

FIGURE 19: Numerical comparison of the horizontal and vertical displacements and angular displacements at the time when the gun ejects the muzzle under the 4 working conditions.

\section{Conclusions}

In this study, the topology optimization problem of the continuum of a base plate under the constraints of quality and engineering was proposed. The modeling method used for the mortar base plate was proven to be accurate and feasible through test verifications. Herein, topology optimization was performed on the structure of a mortar 
base plate using the force transmission path based on the stress analysis of the base plate. The CAE model obtained from the topology optimization was imported into CAD to reconstruct the design and significantly improve design efficiency. The base plate optimization model could satisfy structural stiffness and strength, firing stability, and lightweight design. Compared to the original model, the maximum deformation and stress of the base plate were decreased by $16.85 \%$ and $35.52 \%$, respectively. More importantly, the mass of the optimized base plate was decreased by $12.78 \%$. Thus, this research provides a reference for the performance improvement and structural optimization design of the base plate. At present, the optimization research on the base plate is only in the simulation design stage, and the outline size and quality of the large-caliber mortar base plate are relatively large. How to conduct related impact tests and engineering performance evaluation requires further research.

\section{Data Availability}

The data used to support the findings of this study are included within the article.

\section{Conflicts of Interest}

The authors declare that they have no conflicts of interest.

\section{Acknowledgments}

The authors would like to acknowledge support from the National Natural Science Foundation of China (Grant no. 30107040705).

\section{References}

[1] Z. Tang, Mortar Design, Weapons Industry Press, Beijing, China, 1994.

[2] Y. Shao, Structural Optimization and its Application in Light Artillery Design, Nanjing University of Science and Technology, Nanjing, China, 2013.

[3] P. D. Dunning and H. A. Kim, "Robust topology optimization: minimization of expected and variance of compliance," AIAA Journal, vol. 51, no. 11, pp. 2656-2664, 2013.

[4] H. H. Jang, H. A. Lee, J. Y. Lee, and G. J. Park, "Dynamic response topology optimization in the time domain using equivalent static loads," Aiaa Journal, vol. 50, no. 1, pp. 226-234, 2012.

[5] F. Gomez and B. F. Spencer, "Topology optimization framework for structures subjected to stationary stochastic dynamic loads," Structural and Multidisciplinary Optimization, vol. 59, no. 3, pp. 813-833, 2019.

[6] H.-A. Lee and G.-J. Park, "Nonlinear dynamic response topology optimization using the equivalent static loads method," Computer Methods in Applied Mechanics and Engineering, vol. 283, pp. 956-970, 2015.

[7] L. Zhao, B. Xu, Y. Han, J. Xue, and J. Rong, "Structural topological optimization with dynamic fatigue constraints subject to dynamic random loads," Engineering Structures, vol. 205, no. C, pp. 1-13, Article ID 11089, 2020.

[8] J. Chun, J. Song, and G. H. Paulino, "System-reliability-based design and topology optimization of structures under constraints on first-passage probability," Structural Safety, vol. 76, pp. 81-94, 2019.

[9] K. K. Pydimarry, C. K. Mozumder, N. M. Patel, and J. E. Renaud, "Synthesis of a dynamically loaded structure with topology optimization," SAE International Journal of Passenger Cars-Mechanical Systems, vol. 2, no. 1, pp. 11431150, 2009.

[10] K. A. James, J. S. Hansen, J. R. R. A. Martins, and R. A. Martins, "Structural topology optimization for multiple load cases using a dynamic aggregation technique," Engineering Optimization, vol. 41, no. 12, pp. 1103-1118, 2009.

[11] Z. Luo, J. Yang, L.-P. Chen, Y.-Q. Zhang, and K. Abdel-Malek, "A new hybrid fuzzy-goal programming scheme for multiobjective topological optimization of static and dynamic structures under multiple loading conditions," Structural and Multidisciplinary Optimization, vol. 31, no. 1, pp. 26-39, 2006.

[12] C. Anitescu, E. Atroshchenko, N. Alajlan, and T. Rabczuk, "Artificial neural network methods for the solution of second order boundary value problems," Computers, Materials \& Continua, vol. 59, no. 1, pp. 345-359, 2019.

[13] J. Y. Noh and G. H. Yoon, "Topology optimization of piezoelectric energy harvesting devices considering static and harmonic dynamic loads," Advances in Engineering Software, vol. 53, pp. 45-60, 2012.

[14] K. Yan, G. D. Cheng, and B. P. Wang, “Topology optimization of damping layers in shell structures subject to impact loads for minimum residual vibration," Journal of Sound and Vibration, vol. 431, pp. 226-247, 2018.

[15] K. Dong-Shin, H. K. Lee, and D. J. Hur, "Using topology optimization, light weight design of vehicle mounted voltage converter for impact loading," Journal of the Computational Structural Engineering Institute of Korea, vol. 31, no. 6, pp. 353-358, 2018.

[16] A. Martin and G. D. Gregory, "Structural topology optimization of tall buildings for dynamic seismic excitation using modal decomposition," Engineering Structures, vol. 216, pp. 1-17, Article ID 110717, 2020.

[17] G. Lakshmi Srinivas and J. Arshad, "Topology optimization of rigid-links for industrial manipulator considering dynamic loading conditions," Mechanism and Machine Theory, vol. 153, pp. 1-16, Article ID 103979, 2020.

[18] H. Ghasemi, H. S. Park, and T. Rabczuk, "A multi-material level set-based topology optimization of flexoelectric composites," Computer Methods in Applied Mechanics and Engineering, vol. 332, pp. 47-62, 2018.

[19] H. Ma, Structural Analysis and Optimal Design of Mortar Base Plate, Nanjing University of Science \&Technology, Nanjing, China, 2009.

[20] X. Wang, Structural Optimization Design of a Large Diameter Mortar Base Plate, Nanjing University of Science \& Technology, Nanjing, China, 2018.

[21] X. Zhang, S. Liu, K. Peng et al., "Topological optimization design for mortar's base plate," Journal of Ordnance Equipment Engineering, vol. 37, no. 4, pp. 33-35, 2016.

[22] H. Sayir, C. H. Willison, J. D. Gugliotta et al., "Architecturally design mortar base plate," US, US20110219945, 2011.

[23] F. Niu, Modeling, Solving and Interpretation of Several Problems in Structural Topology Optimization Design, Dalian University of Technology, Dalian, China, 2013.

[24] M. Stolpe and K. Svanberg, "An alternative interpolation scheme for minimum compliance topology optimization," Structural and Multidisciplinary Optimization, vol. 22, no. 2, pp. 116-124, 2001. 